\title{
REMOTE LABORATORIES IN ELECTRONICS ENGINEERING EDUCATION
}

\author{
ZENZEROVIC, P[aolo]; MACUKA, D[aniel] \& MIJAJLOVIC, D[ora]
}

\begin{abstract}
The usage of the remote laboratory as a platform for learning integration in engineering education is discussed in this paper. The concept and partial implementation results of a remote laboratory for the design of automation systems is proposed. Advantages and disadvantages of such a system are elaborated from the didactical, technological and economical point of view and results are presented. An overview of simulation and implementation results is included in the paper. Directions for future work and possible direct applications have also been outlined.
\end{abstract}

Keywords: learning technology, e-learning, engineering education, remote laboratory

\section{INTRODUCTION}

Nowadays, the world has an increasing need for electrical engineers, especially in the field of automation and embedded systems. The teaching facilities, as universities, high schools, institutions for specialized trainings etc. are adapting to that need by increasing the number of places available to study in that area, sometimes neglecting the quality of the educational process. The main problem arising is concerned with the transfer of practical knowledge from the lecturer to the student bearing in mind the existing challenges such as limited time, possession of adequate laboratory space and equipment etc.

At the same time, one of the main megatrends of modern technology is computer integration. There are many outcomes from computer integration such internet, computer aided technology, communications, mobile communications etc. Computer integration is disseminating and infiltrating in many engineering fields, which gives us huge possibilities of acting at a distance. Due to that computer integration motivates and runs the development of many remote learning platforms in all educational fields.

The authors of this paper propose a holistic e-learning remote laboratory platform that can be used in the field of automation system design. The proposed approach is based on the philosophy that universities and other educational facilities should evolve from being institutions that provide instructions to institutions that produce learning [1].

\section{REMOTE LABORATORIES: MAIN OPPORTUNITIES AND CHALLENGES}

With the usage of remote laboratories a lot of solutions to everyday problems can be drawn, but also some challenges arise. A recent study [2] analyzing the impact of new technologies on engineering education, has introduced remote laboratories as a separate technology in the survey. The study results are presented in terms of cross-discipline, cross-country, crosscontinent and cross-technology. This article will present and interpret the results for the disciplines of electronics, electrical and computer engineering and electronics.

Table 1 represents the top three educational technologies used in the field of electronics with no geographical region filtering.

\begin{tabular}{|c|c|}
\hline Technologies & Percentage \\
\hline Simulators & 16,34 \\
\hline Remote labs & 11,11 \\
\hline $\begin{array}{c}\text { E-Learning Platforms and } \\
\text { Architectures }\end{array}$ & 10,46 \\
\hline
\end{tabular}

Tab. 1. Educational technology in electronics - worldwide

Table 2 represents the top three educational technologies used in the field of electronics with geographical restriction to Europe.

\begin{tabular}{|c|c|}
\hline Technologies & Percentage \\
\hline Simulators & 20,00 \\
\hline Remote labs & 15,00 \\
\hline $\begin{array}{c}\text { E-Learning Platforms and } \\
\text { Architectures }\end{array}$ & 13,33 \\
\hline
\end{tabular}

Tab. 2. Educational technology in electronics - Europe

From the above data it is clear that remote laboratories, e-learning platforms and architectures take the second and third place just after simulators. However, if we take in consideration that a remote laboratory in its essence is an e-learning architecture than it is clear that similar solutions take the overall first place.

Table 3 shows the survey results for the impact of remote labs with its major challenges and expected time to adoption. The data is filtered for electrical and computer engineering, electronics and education. The challenges have been numbered as follows [2]:

1 - Better understanding of new ways of interacting with students

\begin{tabular}{|c|c|c|c|c|c|c|c|c|c|c|}
\hline \multirow[t]{2}{*}{ Disciplines } & \multirow{2}{*}{$\begin{array}{c}\text { Votes } \\
(\%)\end{array}$} & \multicolumn{3}{|c|}{ Time to adoption } & \multicolumn{6}{|c|}{ Challenges (\%) } \\
\hline & & $<1$ years & $2-3$ years & $4-5$ years & 1 & 2 & 3 & 4 & 5 & 6 \\
\hline ECE* & 35,58 & 32,43 & 45,95 & 21,62 & 8,11 & 18,92 & 16,22 & 10,81 & 37,84 & 8,11 \\
\hline Electronics & 16,35 & 17,65 & 58,82 & 23,53 & - & 41,18 & 41,18 & - & 11,76 & 5,88 \\
\hline Education & 15,38 & 18,75 & 43,75 & 37,50 & 12,50 & - & 43,75 & 6,25 & 18,75 & 18,75 \\
\hline
\end{tabular}

Tab. 3. Impact of remote labs with its major challenges and expected time to adoption 
2 - Creativity in designing learning experiences

3 - Development of better technology infrastructures

4 - Maturity of the technology

5 - More funds to further development and implantation

6 - New pedagogical methodologies that apply to the technology

Estimated time of two to three years to adoption, across all disciplines, is expected. The major challenges vary considerably between disciplines thus indirectly showing us the current state of the technology. In the field of electrical and computer engineering the major challenge is getting more funds for further development of the technology and to implement the existing conceptual solutions. On the other hand, in the electronics field the major challenge is developing a better technology infrastructure, which will indirectly raise a challenge of getting funds to develop the infrastructure. The educational field shows the same results as before. From the didactical point of view it is interesting that in the first two fields the second major challenge is creativity in designing learning experiences. This challenge may be driven from the fact that the very presence of technology in education does not make it educational. In order to serve for the purpose of education it must have a strong didactical value.

Moreover, Dale's Cone of Experience, mentioned in [3] states that people remember $10 \%$ of what they read, $20 \%$ of what they hear, $30 \%$ of what they see, $50 \%$ of what they hear and see, $70 \%$ of what they say and write, and $90 \%$ of what they say and make while performing a task. Due to that, technology should find a way o overcome this challenge because it has a significant educational potential to simulate reality. This potential is the crucial issue in designing the most important part of the learning process- the experience

As for the creative part of the experience designing, technology should aim at interactive method of learning that will transpose the control of the learning from the teacher to the student through various engaging situations which will allow students to manipulate the content and come up with solutions themselves.

As for the time and resource management a typical problem that should be addressed in most learning facilities is the time usage efficiency of the possessed laboratory equipment. In most of the universities teaching laboratory equipment and laboratory space is shared among more lecturers and students. Mostly, the laboratory spaces are full more than $90 \%$ of the working time of the universities. Of course, not all courses use all of the equipment, but in most cases the equipment that is not used at the course cannot be accessed due to the laboratory space occupation, thus lowering the time usage efficiency of the laboratory equipment. Also, very often, laboratory space is limited, typically being able to accommodate 10 to 15 students at a time. The students take most of the time setting up the experiment they need to perform and a smaller amount of time acquiring the needed data. Taking this fact into account the time usage efficiency of the laboratory equipment is again lowered.

By using remote laboratory platforms those problems could be easily solved since the end user of the platform can access the laboratory equipment from any place at any time using network technologies. Also, the need to use the equipment during the configuration phase of the experiment is eliminated by introducing off-line and online laboratory usage, as well as a dynamic scheduling system for equipment reservation and usage which is incorporated in the platform.

Apart from the typical problems solvable by using remote laboratories a key factor in their usage should be the possibility to easily integrate learning objectives from two or more courses in the engineering curricula.

In general, during the study of engineering sciences lecturers and students are focused on a single technical subject without sufficient cross-disciplinary integration of knowledge and experience [4]. This produces students who generally have very poor ability to integrate theoretical knowledge and practical experience in two or more subject and apply them to real life engineering problems $[5,6]$.

In order for the learning integration to become a feasible model for practical knowledge gaining in engineering education, universities should provide laboratories equipped with different types of equipment (i.e. microcontrollers, digital signal processors, analog signal processing modules, power electronics modules, electro-mechanical actuators, networking interfaces, software development platforms etc.) and give the students the possibility to use them as parts of a single system. It is understandable that with the increasing complexity of the systems that this requirement is almost impossible to meet by the universities. In the area of automation system design the problem can be divided into several sub-problems as follows:

- Provide different system controllers (microcontrollers, programmable logic controllers, digital signal processors etc.)

- Provide different peripheral devices (electromechanical actuators, indicators, input modules, network interfaces, sensors and sensor arrays etc.)

- Provide a system for changing physical interconnections between all modules used in the system (routing of analog and digital signals)

- Provide controlling possibility to manage the system resources

The model of a remote laboratory shown in Figure 1 provides a solution to the postulated problem.

\section{REMOTE LABORATORY HARDWARE}

The above shown remote laboratory consists of a main server computer, a number of different controllers, a number of different peripheral devices, a system for flexible interconnections between the two and a number of remotely accessible equipment.

The main server computer acts as a web server, which enables the end users access to the whole remote laboratory system, and as a system controller for the system. It also runs the end user graphical interface software for system development.

The different controllers and peripherals and their basic necessary hardware which is needed to make them operate are placed in sandboxes. A sandbox represents a minimal group of hardware that an end user can use. 


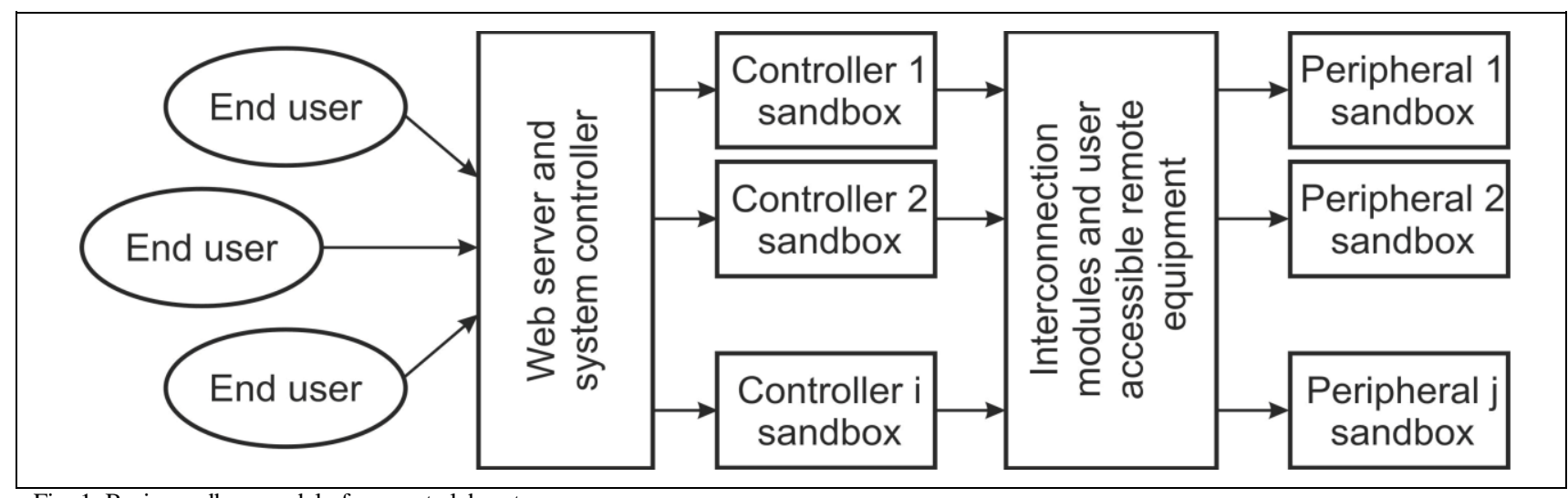

Fig. 1. Basic sandbox model of a remote laboratory

A typical controller sandbox could be a microcontroller, which would consist of the chosen microcontroller, a controllable frequency oscillator and a controllable power supply. A typical peripheral sandbox could be a device for human-machine interfacing, such as an alphanumeric LCD, seven segment displays etc.

In the remote laboratory system there is the possibility of connecting a larger number of controller and peripheral sandboxes, thus enabling the end user to use devices typically considered in different classes in the university curricula.

The key part of the system is the interconnection module. Those are bidirectional switching matrixes that allow the end user to connect one or more of the controller sandboxes to one or more of the peripheral sandboxes. By enabling the end user to do this we have crossed the main challenge of typical university laboratories, we give the students the possibility to use different modules as parts of a single system, and by doing so we have created a basic platform for enabling learning integration in the field of automation system design. This part of the system is called the "Soft-wiring" module and represents the biggest technical and economical challenge in the system realization.

\section{THE SOFT WIRING SYSTEM}

The soft-wiring system at a high abstract level is shown in Figure 2. The system is used to interconnect any/all pins of the first bus to any/all pins of the second bus. This system can be implemented in a few ways, depending on the selection of interconnection switches (switching primitives). The options are switches, relays, multiplexers and demultiplexers, analog multiplexers, crosspoint switches and FPGA technology. A recent study done by Lamza, Zenzerović, Sučić [7] shows the advantages and disadvantages of all the mentioned options for the physical implementation.

The first implemented version of the system [8] based only on microcontrollers and their typical peripheral devices adopt analog multiplexers to interconnect the two busses. This solution was tested and gave positive results. The disadvantage of this solution is the number of integrated circuits needed to interconnect two 32 bit wide busses, and the area needed on PCB boards. The proposed solution uses FPGA devices which minimizes the overall cost and area needed for PCB design. The complete implementation details are given in [7].

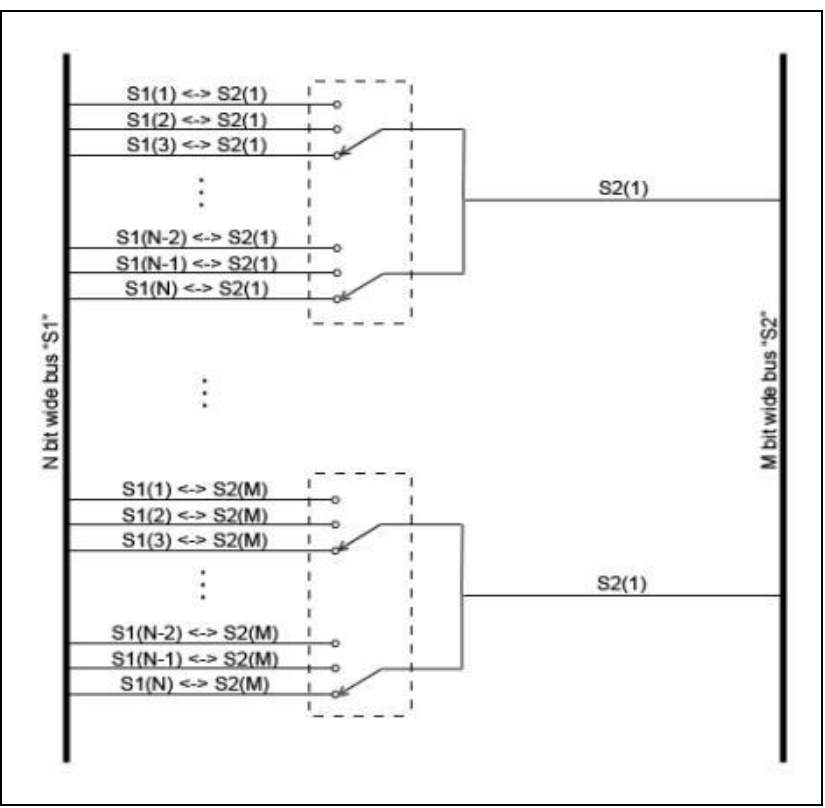

Fig. 2. Softwiring bus model

All implemented solutions of the soft-wiring system rely on pre-defined hardware components. Their architecture cannot be modified by the end user, thus the interconnection module has to be developed so that all the switching components needed to connect any pin of the first bus to any pin of the second bus are present in the system. This is why the topology of the switching primitives network used in all the interconnection modules is complicated and grows in complexity and price significantly with the number of pins of the interconnected busses.

In most of the cases, however, one pin of the first bus, i.e. connected to the end user chosen controller will be connected to one pin of the second bus, i.e. connected to the end user peripheral. This results in very low usage efficiency of the used switching primitives. Nevertheless all interconnections must be possible for the end user. This is why a dynamic soft-wiring system is proposed.

\section{THE DYNAMIC SOFT WIRING SYSTEM}

The dynamic soft-wiring system is implemented in FPGA technology. It allows the end user to connect any/all pins of the two busses in a bi-directional manner. The end must define the two pins that will be connected and the direction of the data flow. The data flow direction can be constant or can dynamically change according to some external signal. 
After the user specification of all the interconnected signals by using the graphical assistant on the remote laboratory web page the data is being compiled into VHDL description language for the FPGA. The generated VHDL code describes the hardware switching components to be implemented. After the generation of the VHDL code, the code is than compiled and programming files are generated with the FPGA programming suite. This system uses Altera FPGA devices and Quartus II programming software for the system implementation. The programming software runs on the web server of the remote laboratory and it is not directly accessible by the end user. The end user does not need to know anything about FPGA technology or VHDL coding to use the system.

The main advantages of such a system is that it creates only the amount of switching primitives that is actually used by the end user, thus lowering drastically the complexity of the interconnection module, making it cheaper to implement. For comparison purposes: the soft-wiring system used about $70 \%$ of the used FPGA while the dynamic soft-wiring solution can be fitted in less than $5 \%$ for a an extensive end user system. This gives the opportunity to use a much cheaper FPGA with less programmable cells, thus reducing the cost of implementation by about 6 to 7 times.

The disadvantage of such a system is the need to have the development studio of the FPGA running on the web server. Also, the programmer for the FPGA device has to be used which adds cost and complexity to the system. Providentially, for teaching purposes a free version of the Altera Quartus II can be used in the system, and programmers and boards can be bought with discounts which makes the dynamic soft-wiring solution the first choice for system implementation.

\section{END USER INTERACTION WITH THE SYSTEM}

Existing solutions in the field of remote laboratories allow their end users to change some variables before the experiment runs at the remote location. This typically includes voltage source parameters changes, current source parameters changes, frequency generators parameters change etc. After the end user has preset the values the experiment can be run. The data is than acquired and sent to the remote end user via the computer network infrastructure. Most of the existing solutions use commercially available generators and measuring equipment with USB, serial or GPIB communication protocol. This is a good but very expensive solution. This is why specialized input and output modules have been developed to be used with the remote laboratory described in this paper. The input modules are divided into two groups: general usage measuring modules and specialized measuring modules. The general usage developing modules are used to acquire data (logical states) of every pin of the controller the user is developing on.

The acquired data can then be displayed in the form of graphical user elements in the web application developed to access the remote laboratory.

The specialized modules were developed in order to acquire data from certain peripherals which need higher sampling frequencies, such as multiplexed seven segment displays, alphanumerical and graphical liquid crystal displays.To remotely control the inputs of the user chosen controller, a specialized remote button module was developed. The module allows the end user to remotely feed inputs to the controller by remotely using pushbuttons or switches to change the input states of the chosen microcontroller pins. In the first implementation of the system [8] all the mentioned modules were developed as stand-alone devices. As the system proposed in this paper uses FPGA technology to implement the soft-wiring modules all the input and output modules described before were implemented as a part of the complete FPGA structure.

\section{ACKNOWLEDGEMENTS}

The authors appreciate the support of the Faculty of Electrical Engineering of the University of Rijeka, the CEEPUS (CIII-HR-0108) network and the Croatian Society for Educational technology in this project.

\section{CONCLUSION}

This paper has presented the concept and implementation of a remote laboratory for the design of automation systems. Both advantages and disadvantages of using remote laboratories for educational purposes have been discussed. From the mentioned recent study remote laboratories should be in use in two to three years, but some general platform improvements need to be done. The authors propose the Soft-wiring and Dynamic Soft-wiring system as a solution for reconfigurability in the end user signal routhing. Partial implementation of the remote laboratory has shown positive results and prooved the concept of those systems. As for the educational perspective the presented remote laboratory platform has the potential to become an interactive method of learning that transposes the control of the learning from the teacher to the student through various engaging situations which will allow students to manipulate the content and come up with solutions themselves.

\section{REFERENCES}

[1] Barr, R. B., Tagg, J.: From teachnig to learning: A new paradigm in undergraduate education, Change, Vol. 27 (1995), No. 6

[2] Martins, S., Meier, R., Castro, M. (Ed).: Engineering Education Report (2012). Retrieved from: http://ohm.ieec.uned.es/eer/

[3] Finnis, A. J.: Learning Technology: Myths and Facts, International Journal of Instructional Technology and Distance Learning, Vol. 1 (2004), No. 5

[4] Pendharkar, G., Judge, S., Gredeskoul, O.: Multidisciplinary Integrative Learning and Assesment in Engineering Education, RMIT University, Melbourne, Australia, 2010

[5] Huber, M., Breen, M.: Integrative Learning: Putting pieces together again, The Carnegie Foundation for the Advancement of Teaching, Stanford, 2007

[6] Ilic, V.: Engineering practice: A drive for curriculum change, Proceedings of the International Conference on Engineering Education, Portugal, 2007

[7] Lamza, S., Zenzerović, P., Sučić, V: Software controlled flexible hardware interconnections: Existing solutions and a novel approach using FPGA technology, Article submitted for publication

[8] Zenzerović, P., Sučić, V.: Remote Laboratory for Microcontroller System Design, Proceedings of the $34^{\text {th }}$ International Convention MIPRO, Opatija, 2011 